\title{
Reflets
}

Revue d'intervention sociale et communautaire

\section{Paternités, du discours aux pratiques}

\section{Madeleine Dubois}

Volume 16, numéro 1, printemps 2010

Paternités méconnues (Deuxième partie)

URI : https://id.erudit.org/iderudit/044440ar

DOI : https://doi.org/10.7202/044440ar

Aller au sommaire du numéro

Éditeur(s)

Reflets : Revue d'intervention sociale et communautaire

ISSN

1203-4576 (imprimé)

1712-8498 (numérique)

Découvrir la revue

Citer ce document

Dubois, M. (2010). Paternités, du discours aux pratiques. Reflets, 16(1), 18-30.

https://doi.org/10.7202/044440ar

Tous droits réservés @ Reflets : Revue d'intervention sociale et communautaire, d'utilisation que vous pouvez consulter en ligne.

https://apropos.erudit.org/fr/usagers/politique-dutilisation/ 


\section{Paternités, du discours aux pratiques}

Tim Paquette est coordonateur sortant de l'Initiative pour l'engagement paternel Réseau ontarien, un réseau ayant récemment pris l'appellation d'Initiative canadienne pour l'engagement des pères (ICEP)/Canadian Father Involvement Initiative (CFII). Créé en 1998, ce réseau cherche à promouvoir l'implication des pères auprès de leurs enfants, par l'éducation, la mobilisation communautaire et la création d'approches et de pratiques novatrices appuyées par des données de recherche probantes.

L'entrevue a été réalisée par Madeleine Dubois.

Reflets : Ayant participé à la création de l'Initiative pour l'engagement paternel Réseau ontarien au cours des années 1997-98, vous y êtes toujours un acteur clé. Quels sont les buts de cette initiative et quels moyens ont été mis en place pour les atteindre?

T. Paquette: Le groupe a pris naissance à Peterborough, avec la publication d'un rapport de recherche mettant en lumière l'importance de l'implication des pères dans le développement de leurs enfants. Le financement de Santé Canada [aujourd'hui l'Agence de santé publique du Canada] a permis de constituer un groupe de personnes intéressées de diverses régions de l'Ontario, incluant des praticiens et des chercheurs, afin de développer notre mission et les principes guidant notre travail. Les activités de l'Initiative pour l'engagement paternel Réseau ontarien s'articulent autour de trois pôles : la création de pratiques porteuses de succès en matière d'intervention avec les pères; la diffusion d'outils de promotion visant à influencer les attitudes de la société face aux rôles des pères et à valoriser leur implication dans la vie de leurs enfants; et la mobilisation communautaire par le biais d'activités d'éducation et de soutien conviviales pour les pères et plus axées sur leurs besoins et leurs défis. 
Par la suite, le projet national intitulé Mon père est important parce que... s'est inspiré du modèle sous-tendant nos activités pour créer un site Internet offrant, notamment, une base de données, une trousse d'outils et des renseignements sur les ressources et programmes destinés aux pères dans l'ensemble du Canada. Ce site loge à l'adresse suivante : www.monpere.ca. D'autres pays se sont aussi intéressés à notre travail et ont reproduit ou traduit nos documents. J'ai d'ailleurs eu l'occasion de me rendre en Australie et au Japon pour travailler avec les représentants gouvernementaux et les groupes qui y font la promotion de notre modèle de mobilisation communautaire face au rôle des pères.

Reflets : Depuis sa création, l'Initiative canadienne pour l'engagement paternel (ICEP) promeut une approche qui intègre la recherche et la pratique, en travaillant en étroite collaboration avec les chercheurs qui s'intéressent aux questions de la paternité.

T. Paquette: En effet, il est essentiel que nos initiatives soient soutenues par des données probantes. Alors que les études sur le rôle maternel s'appuient sur un cursus historiquement dense et toujours en évolution, celles sur le rôle paternel sont plus récentes et se situent à un stade plus embryonnaire. Des études ont néanmoins clairement mis en lumière l'importance du rôle paternel en démontrant les bienfaits pour la santé et le développement optimal de l'enfant associés à l'implication positive du père. L'évolution de notre travail de terrain contribue à enrichir les connaissances dans ce domaine et la recherche se bâtit au fur et à mesure de l'évolution de nos pratiques.

Reflets : Quels sont, selon vous, les changements les plus remarquables dans l'évolution de la paternité et de la situation des pères au cours des dernières années?

T. Paquette : Historiquement, les hommes se définissaient en fonction de leur travail et les femmes, dans la sphère du foyer. Mais contrairement aux femmes, qui se sont battues pour accéder au monde du travail, les hommes n'ont pas revendiqué, au sein du foyer et de la famille, une place qui reflétait les changements dans les rôles traditionnels homme/femme. Nous sommes néanmoins engagés dans une 
transformation sociale souvent silencieuse, qui amène les hommes à s'impliquer davantage dans l'éducation et les soins prodigués à leurs enfants. Nous constatons ainsi des changements remarquables sur plusieurs plans et nous n'en soulignerons ici que quelques-uns.

Le rôle du père comme pourvoyeur de soins est de plus en plus reconnu, ce qui se reflète dans les données de recherche révélant une plus grande implication des pères dans les soins prodigués à leurs enfants. L'étude de Hoffman and Ravanera (2007) a démontré qu'en 1970, les pères consacraient $40 \%$ du temps dédié par les mères aux soins des enfants, alors qu'au milieu des années 1990, ce pourcentage est passé à $67 \%$ pour les pères.

Le pourcentage de pères s'étant prévalus de congés parentaux a triplé au cours de la dernière décennie, se situant, selon les données de Statistique Canada de 2008, à $12 \%$ au Canada et à plus de $56 \%$ au Québec. De surcroît, entre 51 et $55 \%$ des pères d'enfants nés au cours des années 1990 ont pris une quelconque forme de congé (vacances, heures supplémentaires accumulées, ou autre) suivant la naissance de leurs enfants. Par ailleurs, un nombre croissant de pères font usage de politiques en milieu de travail telles que les heures plus souples, le travail à temps partiel ou le télétravail afin de mieux assumer des tâches d'éducation et de soin auprès de leurs enfants. De plus, depuis dix ans, on note une augmentation sensible de la moyenne de jours d'absence du travail (de 1,8 à 6,3 journées par année) attribués par les pères aux soins requis par leurs enfants d'âge préscolaire.

Également, les tribunaux de la famille reconnaissent davantage l'apport des pères comme pourvoyeurs de soins, ce qui résulte en une augmentation des situations de gardes partagées et du nombre de pères chefs de familles.Ainsi, selon les données du Recensement de 2006, au Canada et en Ontario, le pourcentage de pères chefs de familles monoparentales s'élève à $20 \%$ de l'ensemble de ces familles, un taux augmentant deux fois plus rapidement que pour les mères. Confrontés à ce nouveau phénomène, de nombreux organismes de santé et de services sociaux qui travaillent avec les familles semblent néanmoins peu préparés à y répondre de façon adéquate. 
Reflets : L'évolution de la paternité s'accompagne de défis pour les pères, pour les familles et pour la société. Quels sont actuellement les principaux défis auxquels sont confrontés les hommes dans leur rôle de père et quels sont les enjeux sociaux qui y sont associés?

T. Paquette: En dépit de changements dans les rôles sociaux pour les hommes et les femmes, les garçons grandissent souvent sans renforcement de leurs capacités de prendre soin de quelqu'un. La socialisation qu'ils reçoivent favorise peu le développement d'habiletés liées à la dispensation de soins, à l'expression de l'affection, à la capacité d'empathie et elle valorise encore la force, la performance. Comme leur socialisation les prépare peu à la nouvelle paternité, il arrive qu'en devenant pères, les hommes aient à faire beaucoup d'apprentissages. Pour les femmes, ce chemin vers la maternité est mieux reconnu, balisé depuis de nombreux siècles. On a donc énormément de travail à faire pour mieux outiller les garçons et les hommes à assumer les rôles dorénavant attendus d'eux.

Par ailleurs, malgré l'augmentation importante du temps de soins accordé à leurs enfants, les pères font encore face à des attentes sociales contradictoires. Ils sont encouragés à s'impliquer, mais sans trop modifier l'équilibre familial, à se libérer des rôles traditionnels de père, tout en maintenant une image de virilité, à assumer davantage de responsabilités pour les soins aux enfants, sans toutefois apporter des changements aux façons de faire établies. Les contradictions sont aussi encore très présentes dans le monde du travail. Comme le révèle l'Étude nationale sur le conflit entre le travail et la vie personnelle menée par Duxbury et Higgins en 2001, les employeurs ont des attentes plus élevées face au nombre d'heures de travail pour les hommes et sont moins prêts à leur accorder du temps pour s'acquitter de responsabilités familiales. Ces exemples témoignent de la tension émanant de la nécessité, pour les hommes, d'être un bon pourvoyeur, de répondre aux besoins des employeurs et d'être engagés auprès des enfants.

Un autre défi, et non le moindre, émane du fait que les modèles et les images qui sont véhiculés au sujet des hommes et des pères au sein de notre société sont plutôt négatifs. Il s'agit en effet d'une importante constatation mise en lumière par les membres 
du réseau affiliés à notre projet national sur la paternité, et cela a mené à la création d'une campagne médiatique nationale visant à offrir une image plus réaliste et plus positive du rôle paternel.

Reflets : D'après votre expérience et vos observations, quelle place est-elle faite aux hommes, et plus particulièrement aux pères, dans les services sociaux et de la santé?

T. Paquette : Malgré les percées vers une meilleure reconnaissance de la place des pères auprès de leurs enfants et en dépit des nombreuses données soulignant l'importance de leur implication comme déterminant de santé chez les enfants et les adolescents, les services, les programmes et les politiques sociales visant à favoriser une meilleure santé physique, psychologique et économique chez les enfants ne dirigent généralement leurs interventions que vers les mères. Un trop grand nombre de ces programmes ne font pas de place à l'implication des pères et ne tiennent pas compte de leurs besoins, ni de ceux des enfants en ce qui a trait à l'implication paternelle. Les récentes initiatives ayant pour objectif de remédier à ces manques se retrouvent souvent peu ou mal financées par les gouvernements, même si les quelques initiatives communautaires dans le domaine de la paternité connaissent une augmentation de la participation.

Par ailleurs, plusieurs services s'adressant aux hommes et aux pères sont encore conçus en fonction d'une approche déficitaire, c'est-à-dire que les organismes ont tendance à offrir aux pères des services et des ressources centrés sur les lacunes qui leur sont attribuées plutôt que sur le travail avec leurs aptitudes et leurs forces. C'est comme si les pères cherchant à obtenir des services étaient perçus ou classés dans une des catégories suivantes : pères abuseurs, pères ayant des inaptitudes affectives ou sur le plan de l'expression d'émotions, pères insuffisamment impliqués dans les tâches domestiques ou dans les soins aux enfants, pour n'en nommer que quelques-unes des plus répandues. Ancrées dans une vision étroite de ce que doit être un bon parent, les interventions basées sur des approches déficitaires sont inefficaces, non seulement parce qu'elles occultent le progrès et le cheminement des pères, mais aussi parce qu'elles créent des barrières plutôt que de promouvoir le changement. 
Les professionnels qui communiquent avec les parents le font souvent uniquement avec la mère plutôt que de tenter d'impliquer les deux parents, perpétuant ainsi le mythe que ce rôle de responsables des soins aux enfants appartient surtout à la mère et que les pères s'y intéressent peu. Dans les services en matière de bien-être à l'enfance, le dossier familial est souvent au nom de la mère, même lorsque le père est le principal pourvoyeur de soins.

Au Canada, au cours des cinquante dernières années, nous avons développé une panoplie d'initiatives et de ressources s'adressant aux mères et aux enfants. Il s'avère maintenant nécessaire de renouveler certaines de ces pratiques afin de les rendre plus inclusives pour les pères, en créant et en assurant la mise en place d'activités, de ressources, de services et de programmes leur étant adressés.

Reflets: Un des objectifs de l'Initiative canadienne pour l'engagement paternel est justement de développer et de faire la promotion d'approches d'intervention plus susceptibles d'engager les pères et de répondre plus adéquatement aux besoins qu'ils expriment. Quelles seraient les assises et les caractéristiques de telles pratiques et approches?

T. Paquette : Elles s'appuieraient tout d'abord sur la prémisse que la responsabilité d'éduquer et de prendre soin des enfants est de plus en plus partagée par les deux parents. Elles favoriseraient l'implication des pères en valorisant l'importance de leur contribution au bien-être de leurs enfants et de la famille et en mettant l'accent sur leurs forces et sur leur apport positif.

Une approche d'intervention auprès des pères centrée sur les forces reconnait qu'ils ont les aptitudes et la volonté de s'engager auprès de leurs enfants, de leur prodiguer des soins, de comprendre les besoins uniques à chacun d'entre eux et de s'adapter aux changements à mesure qu'ils grandissent, de fournir des ressources pour le bien-être matériel de leurs enfants, de créer avec eux des liens d'attachement sains et durables, favorisant le partage et la communication. 
Reflets : Grâce au travail de l'ICEP, mais aussi aux initiatives d'organismes et d'intervenants, plusieurs programmes novateurs s'adressant aux pères ou s'intéressant plus spécifiquement à leurs besoins et réalités ont été créés au cours des dernières années. Pouvez-vous donner des exemples de tels programmes, des objectifs qu'ils visent et des moyens pris pour y parvenir?

T. Paquette: Voici des exemples de quelques programmes que je connais davantage.

À Ottawa, le Centre Youville, qui offre depuis plusieurs années des services pour les jeunes mères et leurs enfants, a commencé récemment à offrir aux jeunes pères un programme de soutien dans divers domaines. Ce programme a évolué et s'est enrichi au fil des années, à mesure que se définissaient les besoins des jeunes pères. Il a commencé par des soirées ouvertes leur offrant un repas, l'accès au gymnase et à une banque alimentaire, pour ensuite mettre sur pied des cours menant à l'obtention d'un diplôme d'études secondaires, du soutien pour la recherche d'emploi et le développement d'habiletés parentales et diverses activités pour promouvoir les relations entre le père et son enfant. En élargissant ainsi sa gamme de services, le Centre était en mesure d'aider les parents, mères et pères, en tant que jeunes familles.

Également à Ottawa, la Société d'aide à l'enfance initie des pratiques axées sur les forces de la famille et du père et travaille à mieux engager les pères en leur offrant de l'aide individuelle et des groupes de soutien.

À Abbotsford en Colombie-Britannique, un programme situé dans une école secondaire aide d'abord les jeunes pères à compléter les cours nécessaires à l'obtention d'un diplôme d'études, mais il leur offre également du soutien pour qu'ils assument mieux leur rôle de parent, ainsi que de la sensibilisation sur divers sujets tels que les relations amoureuses, des questions légales ou ayant trait à la garde d'enfants.

On retrouve également de plus en plus de cours de parentage pour les pères qui sont animés par des pères, ainsi que des publications 
dirigées vers les pères. L'Initiative canadienne pour l'engagement paternel a publié des livrets sur une variété de sujets qui sont distribués par les organismes de santé publique, mais qui sont aussi disponibles sur son site Internet, à l'adresse www.cfii.ca.

Reflets : Comme les programmes et ressources s'adressant aux hommes sont plus récents, moins connus et que, par ailleurs, ces derniers ont peut-être des réticences à participer, il faut aussi réfléchir aux stratégies pour les rejoindre et les inciter à y prendre part. Quels sont certains des moyens mis en place pour encourager la participation des pères?

T. Paquette : De nombreux facteurs contribuent aux réticences observées chez les hommes lorsqu'il s'agit de participer à des programmes leur étant destinés et les efforts visant à encourager leur participation doivent en tenir compte. Soulignons, entre autres, que les hommes ont souvent des réseaux différents ou plus limités que ceux des femmes et qu'ils sont toujours aujourd'hui confrontés à des mythes persistants au sujet de la faiblesse de l'homme demandant du soutien. S'ajoute à ces facteurs la perception que les centres de santé communautaires, les ressources pour parents ou les centres de la petite enfance sont des services pour les femmes et les mères et qu'ils ne s'adressent donc pas aux pères.

Par ailleurs, les hommes ont sans doute moins de pression sociale les incitant à s'impliquer davantage, sans compter que la culture de travail est moins tolérante face à l'absentéisme d'un homme pour des raisons familiales. Il faut aussi reconnaitre que parfois, les mères manifestent une certaine ambivalence face à une plus grande implication paternelle, soit parce qu'elles tiennent à maintenir la responsabilité d'un domaine qu'elles perçoivent comme étant le leur ou qu'elles ont des préoccupations face à la compétence du père comme pourvoyeur de soins aux enfants, ou pour toute autre raison. Les hommes peuvent donc hésiter à s'impliquer afin de ne pas bousculer les dynamiques familiales.

Les approches destinées aux pères étant relativement récentes, les façons de rejoindre ces derniers sont moins connues. Il faut 
donc que les pourvoyeurs de services continuent d'explorer ce qui semble donner de meilleurs résultats et partagent leurs observations à ce sujet. On a constaté, par exemple, qu'on peut rejoindre les hommes dans leurs milieux de travail en organisant, sur l'heure du dîner, des groupes de discussion ou des ateliers sur divers sujets associés aux rôles des pères. Qu'ils soient offerts dans les milieux de travail ou ailleurs, il est important que les programmes destinés aux hommes aient des noms susceptibles de les accrocher et de les intéresser et que les groupes soient animés par des pères.

Plusieurs stratégies pour rejoindre les pères ont été développées dans le cadre de projets sur l'engagement paternel financés par l'Agence de santé publique du Canada. Les projets intitulés Sur le terrain des pères et Mon père est important parce que... ne sont que deux exemples d'excellentes ressources offrant des outils à cet effet.

Le travail effectué dans le cadre de l'Initiative canadienne pour l'engagement paternel nous a aussi permis d'observer que les pères sont plus aptes à faire appel aux services lorsqu'ils ont besoin d'appui face à une crise qui concerne leurs enfants. C'est souvent par le biais du bien-être de l'enfant qu'on peut rejoindre les pères. Ils participent donc plus volontiers lorsque les services offrent des ressources traitant des habiletés parentales ou de la communication avec leurs enfants ou des activités, sportives ou ludiques, leur permettant de bénéficier d'un environnement stimulant et agréable avec leurs enfants.

Reflets : Les défis sont donc nombreux pour les organismes qui cherchent à encourager la participation des pères. Mais les lacunes ou retards dans l'offre de ressources ou de services aux hommes et aux pères s'expliquent sans doute aussi par certaines lacunes ou des manques de sensibilisation de la part des organismes et des bailleurs de fonds. Quelles lacunes ont été relevées au sein de ces instances?

T. Paquette: Il est d'abord essentiel que les organismes affichent clairement leur positionnement en faveur de l'implication paternelle et qu'ils développent des façons de faire et des programmes congruents 
avec celui-ci. Il ne suffit pas de diriger les pères vers un groupe ou un service leur étant destiné, il faut aussi que l'organisme dans son ensemble manifeste son soutien face à l'implication paternelle, en fasse la promotion et persiste dans ses efforts pour offrir aux pères des programmes et un appui significatifs.

Quant aux intervenantes et aux intervenants œuvrant au sein d'organismes de services aux familles, ils doivent réfléchir à leurs attitudes et aux valeurs qu'ils véhiculent face aux rôles parentaux, à leurs partis pris face aux rapports sociaux de sexe. Est-ce que dans nos pratiques on tente d'aider les mères que l'on juge moins compétentes, mais on exclut les pères jugés incompétents d'une façon ou d'une autre? Nos pratiques offrent-elles la chance aux pères en difficultés de s'améliorer et d'être plus présents auprès de leurs enfants? La participation à des réseaux regroupant des intervenants de divers organismes soucieux de mieux répondre aux besoins des pères peut faciliter la réflexion sur les pratiques existantes et contribuer à y intégrer des éléments plus inclusifs pour ces derniers.

Il me semble également important de souligner que la promotion de l'implication paternelle ne doit pas être associée avec les mouvements de revendication des droits des hommes. Les initiatives de promotion de l'implication paternelle ont uniquement comme but de maximiser le bien-être des enfants et des familles, et elles sont soutenues par des données de recherche démontrant sans équivoque que l'implication positive des pères contribue à ce bien-être et au sain développement des enfants.

Par contre, les gouvernements et les bailleurs de fonds manifestent peu d'intérêt face à l'importance du rôle paternel et à sa promotion. Ils offrent peu d'incitatifs aux organismes pour qu'ils développent des pratiques plus inclusives et mieux ciblées pour les pères, puisque peu de fonds y sont rattachés. Le succès des programmes est souvent lié au nombre de mères et d'enfants qui y participent, ce qui constitue une autre façon de faire abstraction de l'importance du rôle des pères. En tant que société, nous devons faire la promotion de l'importance de ce rôle et en assurer le rayonnement parce que les enfants ont besoin de leurs pères, 
tout comme ces derniers bénéficient en tant qu'êtres humains du contact soutenu avec leurs enfants.

Reflets: Vu les contraintes budgétaires auxquelles ils sont astreints, les organismes de services aux familles se voient sans doute confrontés au défi de tenir compte des besoins des pères face aux services sociaux et de santé sans pour autant négliger les préoccupations sur l'aide dont les mères ont également besoin. Comment les organismes peuvent-ils répondre à ce souci?

T. Paquette: Il ne s'agit pas ici de réduire les services offerts aux mères ou encore de dédoubler les services en divisant le couple parental, mais plutôt d'offrir un soutien adapté aux pères dont pourront aussi bénéficier les mères et leurs enfants. On pourrait donc demander aux pères d'abord, mais aussi à l'ensemble de la famille, quels sont leurs besoins. Pourquoi ne pas inclure les mères dans le processus, notamment en leur permettant d'expérimenter en quoi l'implication du père peut être bénéfique pour elles? Nous savons par exemple que plus un père est impliqué dans la vie de son enfant, plus il sera porté à contribuer financièrement au bien-être de la famille, ce qui réduit les risques de pauvreté et de dépression chez les mères monoparentales.

Les mères comme les pères apportent beaucoup à leurs enfants, chacun à leur manière. Bien que la contribution de chacun soit souvent différente et parfois inégale, elle est néanmoins importante et nécessaire au bon développement des enfants. C'est cette différence qu'il faut mettre à profit. En écoutant les besoins des pères et en amenant les mères à comprendre en quoi cette spécificité est bénéfique, et donc à respecter les différences dans les méthodes d'éducation du père, on arrivera à offrir des services aux pères sans nier les besoins de mères.

Donc, les moyens à utiliser pour tenir compte des besoins des hommes sans nier ceux des femmes seraient de faire prendre conscience, autant aux mères qu'aux acteurs liés aux services sociaux, qu'écouter les hommes et leur laisser de la place pour expérimenter leur rôle de père, à leur manière, ne peut qu'être bénéfique pour la famille. Pour ce faire, il s'agit simplement de 
sensibiliser, d'éduquer l'ensemble de la population ainsi que les prestataires de services quant aux bienfaits de l'implication paternelle et de l'expérimenter, notamment en mettant en place des projets pilotes et des initiatives communautaires qui rejoignent les pères.

Reflets: Dans l'état actuel des rapports hommes-femmes et pères-mères, quelle place faisons-nous aux enfants?

T. Paquette: Il n'est pas question ici des rapports hommes-femmes ou pèresmères, mais plutôt du rapport couple parental-enfants, sans égard à l'état matrimonial des parents. Sachant que les rapports hommesfemmes tendent à être plus égalitaires quand il y a une plus grande implication de la part du père auprès des enfants comme dans la vie familiale, il devient évident qu'il faut faire une place au père dans l'exercice de la parentalité.

Généralement, les pères qui demandent de l'aide ou qui ressentent un malaise quant à leur implication parentale le font pour le bienêtre de leurs enfants.Autant pour les mères que pour les pères, les enfants sont un puissant moteur de changement. Plus que jamais, considérant la charge de travail qui pèse sur les épaules des deux parents, il est important que les parents fassent équipe afin de conserver cette place centrale des enfants au sein de la famille.

Reflets : Existe-t-il des résultats d'études ou des données de recherches qui pourraient orienter les services ou les organismes désirant offrir des ressources aux pères, qui présentent, notamment, des exemples de pratiques réussies dans ce domaine?

T. Paquette: Dans leurs rapports, les deux projets auxquels nous avons fait référence précédemment, soit Sur le terrain des pères et Mon père est important parce que... présentent des boîtes à outils pour les organismes qui cherchent à offrir des services plus conviviaux pour les pères. L'ICEP offre aussi aux organismes et aux communautés qui désirent mettre en place des initiatives proactives pour susciter l'engagement paternel des sessions de développement communautaire comportant notamment la présentation de données de recherche sur les effets de l'implication paternelle 
pour les enfants et les familles, une trousse d'outils, des suggestions d'activités de mobilisation et de sensibilisation s'adressant à l'ensemble de la communauté.

Reflets : Une dernière réflexion avant de clore cette entrevue?

T. Paquette: J'aimerais réitérer l'importance de mieux définir le rôle du père dans la société d'aujourd'hui. Le discours sur les rôles parentaux est encore très axé sur l'apport de la mère, alors que celui du père prend de plus en plus d'importance dans la famille actuelle. Il doit être davantage mis en lumière et valorisé. Une meilleure articulation du rôle paternel est essentielle si, comme société, nous prenons au sérieux la responsabilité de mieux éduquer nos garçons, afin de les préparer à assumer les nouveaux rôles qui leur incomberont comme hommes et comme pères. 